\title{
On some inequalities for generalized s-convex functions and applications on fractal sets
}

\author{
Adem Kiliçman $^{\mathrm{a}, *}$, Wedad Saleh ${ }^{\mathrm{b}}$ \\ ${ }^{a}$ Department of Mathematics and Institute for Mathematical Research, University Putra Malaysia, 43400 UPM, Serdang, Selangor, \\ Malaysia. \\ ${ }^{b}$ Department of Mathematics, Putra University of Malaysia (UPM), Serdang, Malaysia.
}

Communicated by A. Atangana

\begin{abstract}
The authors present some new inequalities of generalized Hermite-Hadamard's type for the class of functions whose second local fractional derivatives of order $\alpha$ in absolute value at certain powers are generalized s-convex functions in the second sense. Moreover, some applications are given. (c)2017 all rights reserved.
\end{abstract}

Keywords: s-convex functions, fractal space, local fractional derivative.

2010 MSC: 26A51, 26D07, 26D15.

\section{Introduction and preliminaries}

Let $f: U \subset \mathbb{R} \longrightarrow \mathbb{R}^{\alpha}$. For any $x_{1}, x_{2} \in U$ and $\gamma \in[0,1]$ if the inequality

$$
f\left(\gamma x_{1}+(1-\gamma) x_{2}\right) \leqslant \gamma^{\alpha} f\left(x_{1}\right)+(1-\gamma)^{\alpha} f\left(x_{2}\right)
$$

holds, then $\mathrm{f}$ is called a generalized convex function on $\mathrm{U}$ [14]. In $\alpha=1$, we have convex function, which means that if $P_{1}, P_{2}$, and $P_{3}$ are three distinct points on the graph of $f$ with $P_{2}$ between $P_{1}$ and $P_{3}$, then $P_{2}$ is on or below the chord $P_{1} P_{3}$ [7].

The convexity of functions plays a significant role in many fields, for example, in biological system, economy, optimization, and so on [6, 17].

Let $f \in{ }_{a_{1}} I_{a_{2}}^{(\alpha)}$ be a generalized convex function on $\left[a_{1}, a_{2}\right]$ with $a_{1}<a_{2}$. Then,

$$
f\left(\frac{a_{1}+a_{2}}{2}\right) \leqslant \frac{\Gamma(1+\alpha)}{\left(a_{2}-a_{1}\right)^{\alpha}} \quad a_{1} I_{a_{2}}^{(\alpha)} f(x) \leqslant \frac{f\left(a_{1}\right)+f\left(a_{2}\right)}{2^{\alpha}} .
$$

is known as generalized Hermite-Hadmard's inequality [14]. Many authers paid attention to the study of

\footnotetext{
*Corresponding author

Email addresses: akilic@upm.edu.my (Adem Kiliçman), wed_10_777@hotmail.com (Wedad Saleh)
} doi:10.22436/jnsa.010.02.22 
generalized Hermite-Hadmard's inequality and generalized convex function, see [9, 13]. If $\alpha=1$ in $(1.1)$, then [5]

$$
f\left(\frac{a_{1}+a_{2}}{2}\right) \leqslant \frac{1}{a_{2}-a_{1}} \int_{a_{1}}^{a_{2}} f(x) d x \leqslant \frac{f\left(a_{1}\right)+f\left(a_{2}\right)}{2},
$$

which is known as classical Hermite-Hadamard inequality, for more properties about this inequality we refer the interested readers to $[4,8]$.

In [12], Mo and Sui introduced the definitions of two kinds of generalized s-convex functions on fractal sets such as follows.

\section{Definition 1.1.}

(i) A function $\mathrm{f}: \mathbb{R}_{+} \longrightarrow \mathbb{R}^{\alpha}$, is called a generalized s-convex $(0<\mathrm{s}<1)$ in the first sense if

$$
f\left(\gamma_{1} x_{1}+\gamma_{2} x_{2}\right) \leqslant \gamma_{1}^{s \alpha} f\left(x_{1}\right)+\gamma_{2}^{s \alpha} f\left(x_{2}\right),
$$

$\forall x_{1}, x_{2} \in \mathbb{R}_{+}, \forall \gamma_{1}, \gamma_{2} \geqslant 0$ with $\gamma_{1}^{s}+\gamma_{2}^{s}=1$.

(ii) A function $f: \mathbb{R}_{+} \longrightarrow \mathbb{R}^{\alpha}$, is called a generalized s-convex $(0<s<1)$ in the second sense if (1.2) holds $\forall x_{1}, x_{2} \in \mathbb{R}_{+}, \forall \gamma_{1}, \gamma_{2} \geqslant 0$ with $\gamma_{1}+\gamma_{2}=1$.

In the same paper, [12], Mo and Sui proved that all functions which are generalized s-convex in the second sense, for $s \in(0,1)$, are non-negative.

If $\alpha=1$ in Definition 1.1, then we have the classical s-convex functions in the first sense (second sense) see $[5]$.

Also, in [5], Dragomir and Fitzatrick demonstrated a variation of Hadamard's inequality which holds for s-convex functions in the second sense.

Theorem 1.2. Assume that $\mathrm{f}: \mathbb{R}_{+} \longrightarrow \mathbb{R}_{+}$is a s-convex function in the second sense, $0<\mathrm{s}<1$ and $\mathrm{a}_{1}, \mathrm{a}_{2} \in \mathbb{R}_{+}$, $\mathrm{a}_{1}<\mathrm{a}_{2}$. If $\mathrm{f} \in \mathrm{L}^{1}\left(\left[\mathrm{a}_{1}, \mathrm{a}_{2}\right]\right)$, then

$$
2^{s-1} f\left(\frac{a_{1}+a_{2}}{2}\right) \leqslant \frac{1}{a_{2}-a_{1}} \int_{a_{1}}^{a_{2}} f(x) d x \leqslant \frac{f\left(a_{1}\right)+f\left(a_{2}\right)}{s+1} .
$$

If we set $c=\frac{1}{s+1}$, then it is the best possible in the second inequality in (1.3).

A variation of generalized Hadamard's inequality holds for generalized s-convex functions in the second sense.

Theorem 1.3. Assume that $\mathrm{f}: \mathbb{R}_{+} \longrightarrow \mathbb{R}_{+}^{\alpha}$ is a generalized s-convex function in the second sense where $0<\mathrm{s}<1$ and $\mathrm{a}_{1}, \mathrm{a}_{2} \in \mathbb{R}_{+}$with $\mathrm{a}_{1}<\mathrm{a}_{2}$. If $\mathrm{f} \in \mathrm{L}^{1}\left(\left[\mathrm{a}_{1}, \mathrm{a}_{2}\right]\right)$, then

$$
2^{\alpha(s-1)} f\left(\frac{a_{1}+a_{2}}{2}\right) \leqslant \frac{\Gamma(1+\alpha)}{\left(a_{2}-a_{1}\right)^{\alpha}} \quad a_{1} I_{a_{2}}^{(\alpha)} f(x) \leqslant \frac{\Gamma(1+s \alpha) \Gamma(1+\alpha)}{\Gamma(1+(s+1) \alpha)}\left(f\left(a_{1}\right)+f\left(a_{2}\right)\right) .
$$

If we set $c=\frac{\Gamma(1+s \alpha) \Gamma(1+\alpha)}{\Gamma(1+(s+1) \alpha)}$ for $s \in(0,1)$, then it is the best possible in the second inequality (1.4), the proof was started by Kiliçman and Saleh [10].

There are many researchers studied the properties of functions on fractal space and constructed many kinds of fractional calculus by using different approaches; see [3, 18, 21].

This work is organized as follows. After this introduction, in Section 2, we discuss some generalized Hermite-Hadamard type inequalities for the class of functions whose second local fractional derivatives in absolute value at certain powers are generalized s-convex functions in the second sense. Finally, some applications of the results from Section 2 for special mean are considered. 


\section{Main results}

We need the following lemma to prove our main results:

Lemma 2.1. Assume that $\mathrm{f}:\left[\mathrm{a}_{1}, \mathrm{a}_{2}\right] \subset \mathbb{R} \longrightarrow \mathbb{R}^{\alpha}$ is a local fractional derivative of order $\alpha\left(\mathrm{f} \in \mathrm{D}_{\alpha}\right)$ on $\left(\mathrm{a}_{1}, \mathrm{a}_{2}\right)$ with $\mathrm{a}_{1}<\mathrm{a}_{2}$. If $\mathrm{f}^{(2 \alpha)} \in \mathrm{C}_{\alpha}\left[\mathrm{a}_{1}, \mathrm{a}_{2}\right]$, then the following equality holds:

$$
\begin{gathered}
\frac{\Gamma(1+2 \alpha)[\Gamma(1+\alpha)]^{2}}{2^{\alpha}\left(a_{2}-a_{1}\right)^{\alpha}} a_{1} I_{a_{2}}^{(\alpha)} f(x)-\frac{\Gamma(1+2 \alpha)}{2^{\alpha}} f\left(\frac{a_{1}+a_{2}}{2}\right) \\
=\frac{\left(a_{2}-a_{1}\right)^{2 \alpha}}{16^{\alpha}}\left[{ }_{0} I_{1}^{(\alpha)} \gamma^{2 \alpha} f^{(2 \alpha)}\left(\gamma \frac{a_{1}+a_{2}}{2}+(1-\gamma) a_{1}\right)\right. \\
\left.\quad+{ }_{0} I_{1}^{(\alpha)}(\gamma-1)^{2 \alpha_{f}}{ }^{(2 \alpha)}\left(\gamma a_{2}+(1-\gamma) \frac{a_{1}+a_{2}}{2}\right)\right] .
\end{gathered}
$$

Proof. From the local fractional integration by parts, we get

$$
\begin{aligned}
A_{1}= & \frac{1}{\Gamma(1+\alpha)} \int_{0}^{1} \gamma^{2 \alpha_{f}(2 \alpha)}\left(\gamma \frac{a_{1}+a_{2}}{2}+(1-\gamma) a_{1}\right)(d \gamma)^{\alpha} \\
= & \left(\frac{2}{a_{2}-a_{1}}\right)^{\alpha} f^{(\alpha)}\left(\frac{a_{1}+a_{2}}{2}\right)-\left.\Gamma(1+2 \alpha)\left(\frac{2}{a_{2}-a_{1}}\right)^{2 \alpha} \gamma^{\alpha} f\left(\gamma \frac{a_{1}+a_{2}}{2}+(1-\gamma) a_{1}\right)\right|_{0} ^{1} \\
& +\Gamma(1+2 \alpha) \Gamma(1+\alpha)\left(\frac{2}{b-a}\right)^{2 \alpha} \int_{0}^{1} f\left(\gamma \frac{a_{1}+a_{2}}{2}+(1-\gamma) a_{1}\right)(d \gamma)^{\alpha} \\
= & \left(\frac{2}{a_{2}-a_{1}}\right)^{\alpha} f^{(\alpha)}\left(\frac{a_{1}+a_{2}}{2}\right)-\Gamma(1+2 \alpha)\left(\frac{2}{a_{2}-a_{1}}\right)^{2 \alpha} f\left(\frac{a_{1}+a_{2}}{2}\right) \\
& +\Gamma(1+2 \alpha) \Gamma(1+\alpha)\left(\frac{2}{b-a}\right)^{2 \alpha} \int_{0}^{1} f\left(\gamma \frac{a_{1}+a_{2}}{2}+(1-\gamma) a_{1}\right)(d \gamma)^{\alpha} .
\end{aligned}
$$

Setting $x=\gamma \frac{a_{1}+a_{2}}{2}+(1-\gamma) a_{1}$, for $\gamma \in[0,1]$ and multiplying both sides in the last equation by $\frac{\left(a_{2}-a_{1}\right)^{2 \alpha}}{16^{\alpha}}$, we get

$$
\begin{aligned}
A_{1} & =\frac{\left(a_{2}-a_{1}\right)^{2 \alpha}}{16^{\alpha}} I_{1}^{(\alpha)} \gamma^{2 \alpha} f^{(2 \alpha)}\left(\gamma \frac{a_{1}+a_{2}}{2}+(1-\gamma) a_{1}\right) \\
& =\frac{\left(a_{2}-a_{1}\right)^{\alpha}}{8^{\alpha}} f^{(\alpha)}\left(\frac{a_{1}+a_{2}}{2}\right)-\frac{\Gamma(1+2 \alpha)}{4^{\alpha}} f\left(\frac{a_{1}+a_{2}}{2}\right)+\frac{\Gamma(1+2 \alpha) \Gamma(1+\alpha)}{2^{\alpha}\left(a_{2}-a_{1}\right)^{\alpha}} \int_{a_{1}}^{\frac{a_{1}+a_{2}}{2}} f(x)(d x)^{\alpha} .
\end{aligned}
$$

By the similar way, also we have

$$
\begin{aligned}
A_{2} & =\frac{\left(a_{2}-a_{1}\right)^{2 \alpha}}{16^{\alpha}} I_{1}^{(\alpha)}(\gamma-1)^{2 \alpha} f^{(2 \alpha)}\left(\gamma a_{2}+(1-\gamma) \frac{a_{1}+a_{2}}{2}\right) \\
& =-\frac{\left(a_{2}-a_{1}\right)^{\alpha}}{8^{\alpha}} f^{(\alpha)}\left(\frac{a_{1}+a_{2}}{2}\right)-\frac{\Gamma(1+2 \alpha)}{4^{\alpha}} f\left(\frac{a_{1}+a_{2}}{2}\right)+\frac{\Gamma(1+2 \alpha) \Gamma(1+\alpha)}{2^{\alpha}\left(a_{2}-a_{1}\right)^{\alpha}} \int_{\frac{a_{1}+a_{2}}{2}}^{a_{2}} f(x)(d x)^{\alpha} .
\end{aligned}
$$

Thus, adding $A_{1}$ and $A_{2}$, we get the desired result.

Theorem 2.2. Assume that $\mathrm{f}: \mathrm{U} \subset[0, \infty) \longrightarrow \mathbb{R}^{\alpha}$ such that $\mathrm{f} \in \mathrm{D}_{\alpha}$ on $\operatorname{Int}(\mathrm{U})$ (Int $(\mathrm{U})$ is the interior of $\mathrm{U}$ ) and $\mathrm{f}^{(2 \alpha)} \in \mathrm{C}_{\alpha}\left[\mathrm{a}_{1}, \mathrm{a}_{2}\right]$, where $\mathrm{a}_{1}, \mathrm{a}_{2} \in \mathrm{U}$ with $\mathrm{a}_{1}<\mathrm{a}_{1}$. If $|\mathrm{f}|$ is generalized $\mathrm{s}$-convex on $\left[\mathrm{a}_{1}, \mathrm{a}_{2}\right]$ for some fixed $0<s \leqslant 1$, then the following inequality holds: 


$$
\begin{aligned}
\mid & \frac{\Gamma(1+2 \alpha)}{2^{\alpha}} f\left(\frac{a_{1}+a_{2}}{2}\right)-\frac{\Gamma(1+2 \alpha)[\Gamma(1+\alpha)]^{2}}{2^{\alpha}\left(a_{2}-a_{1}\right)^{\alpha}} a_{1} I_{a_{2}}^{(\alpha)} f(x) \mid \\
\leqslant & \frac{\left(a_{2}-a_{1}\right)^{2 \alpha}}{16^{\alpha}}\left\{\frac{2^{\alpha} \Gamma(1+(s+2) \alpha)}{\Gamma(1+(s+3) \alpha)}\left|f^{(2 \alpha)}\left(\frac{a_{1}+a_{2}}{2}\right)\right|+\left[\frac{\Gamma(1+s \alpha)}{\Gamma(1+(s+1) \alpha)}\right.\right. \\
& \left.\left.-2^{\alpha} \frac{\Gamma(1+(s+1) \alpha)}{\Gamma(1+(s+2) \alpha)}+\frac{\Gamma(1+(s+2) \alpha)}{\Gamma(1+(s+3) \alpha)}\right]\left[\left|f^{(2 \alpha)}\left(a_{1}\right)\right|+\left|f^{(2 \alpha)}\left(a_{2}\right)\right|\right]\right\} \\
\leqslant & \frac{\left(a_{2}-a_{1}\right)^{2 \alpha}}{16^{\alpha}}\left\{\frac{2^{\alpha(2-s)} \Gamma(1+(s+2) \alpha)}{\Gamma(1+(s+3) \alpha)} \frac{\Gamma(1+s \alpha) \Gamma(1+\alpha)}{\Gamma(1+(s+1) \alpha)}+\frac{\Gamma(1+s \alpha)}{\Gamma(1+(s+1) \alpha)}\right. \\
& \left.-\frac{2^{\alpha} \Gamma(1+(s+1) \alpha)}{\Gamma(1+(s+2) \alpha)}+\frac{\Gamma(1+(s+2) \alpha)}{\Gamma(1+(s+3) \alpha)}\right\}\left[\left|f^{(2 \alpha)}\left(a_{1}\right)\right|+\left|f^{(2 \alpha)}\left(a_{2}\right)\right|\right] .
\end{aligned}
$$

Proof. From Lemma 2.1, we have

$$
\begin{aligned}
&\left|\frac{\Gamma(1+2 \alpha)}{2^{\alpha}} f\left(\frac{a_{1}+a_{2}}{2}\right)-\frac{\Gamma(1+2 \alpha)[\Gamma(1+\alpha)]^{2}}{2^{\alpha}\left(a_{2}-a_{1}\right)^{\alpha}} a_{1} I_{a_{2}}^{(\alpha)} f(x)\right| \\
& \leqslant \frac{\left(a_{2}-a_{1}\right)^{2 \alpha}}{16^{\alpha}}\left[{ }_{0} I_{1}^{(\alpha)} \gamma^{2 \alpha}\left|f^{(2 \alpha)}\left(\gamma \frac{a_{1}+a_{2}}{2}+(1-\gamma) a_{1}\right)\right|\right. \\
&\left.+{ }_{0} I_{1}^{(\alpha)}(\gamma-1)^{2 \alpha}\left|f^{(2 \alpha)}\left(\gamma a_{2}+(1-\gamma) \frac{a_{1}+a_{2}}{2}\right)\right|\right] \\
& \leqslant \frac{\left(a_{2}-a_{1}\right)^{2 \alpha}}{16^{\alpha}}{ }_{0} I_{1}^{(\alpha)} \gamma^{2 \alpha}\left[\gamma^{\alpha s}\left|f^{(2 \alpha)}\left(\frac{a_{1}+a_{2}}{2}\right)\right|+(1-\gamma)^{\alpha s}\left|f^{(2 \alpha)}\left(a_{1}\right)\right|\right] \\
&+\frac{\left(a_{2}-a_{1}\right)^{2 \alpha}}{16^{\alpha}}{ }_{0} I_{1}^{(\alpha)}(\gamma-1)^{2 \alpha}\left[\gamma^{\alpha s}\left|f^{(2 \alpha)}\left(a_{2}\right)\right|+(1-\gamma)^{\alpha s}\left|f^{(2 \alpha)}\left(\frac{a_{1}+a_{2}}{2}\right)\right|\right] \\
&= \frac{\left(a_{2}-a_{1}\right)^{2 \alpha}}{16^{\alpha}}\left\{\frac{\Gamma(1+(s+2) \alpha)}{\Gamma(1+(s+3) \alpha)}\left|f^{(2 \alpha)}\left(\frac{a_{1}+a_{2}}{2}\right)\right|\right. \\
&\left.+\left[\frac{\Gamma(1+\alpha s)}{\Gamma(1+(s+1) \alpha)}-2^{\alpha} \frac{\Gamma(1+(s+1) \alpha)}{\Gamma(1+(s+2) \alpha)}+\frac{\Gamma(1+(s+2) \alpha)}{\Gamma(1+(s+3) \alpha)}\right]\left|f^{(2 \alpha)}\left(a_{1}\right)\right|\right\} \\
&+\frac{\left(a_{2}-a_{1}\right)^{2 \alpha}}{16^{\alpha}}\left\{\frac{\Gamma(1+(s+2) \alpha)}{\Gamma(1+(s+3) \alpha)}\left|f^{(2 \alpha)}\left(\frac{a_{1}+a_{2}}{2}\right)\right|\right. \\
&+\left[\frac{\Gamma(1+\alpha s)}{\Gamma(1+(s+1) \alpha)}-2^{\alpha} \frac{\Gamma(1+(s+1) \alpha)}{\Gamma(1+(s+2) \alpha)}+\frac{\Gamma(1+(s+2) \alpha)}{\Gamma(1+(s+3) \alpha)}\right]\left|f^{(2 \alpha)}\left(a_{2}\right)\right| \\
&\left.+\frac{\Gamma(1+(s+2) \alpha)}{\Gamma(1+(s+3) \alpha)}\left|f^{(2 \alpha)}\left(\frac{a_{1}+a_{2}}{2}\right)\right|\right\} \\
&= \frac{\left(a_{2}-a_{1}\right)^{2 \alpha}}{16^{\alpha}}\left\{\frac{2^{\alpha} \Gamma(1+(s+2) \alpha)}{\Gamma(1+(s+3) \alpha)}\left|f^{(2 \alpha)}\left(\frac{a_{1}+a_{2}}{2}\right)\right|+\left[\frac{\Gamma(1+s \alpha)}{\Gamma(1+(s+1) \alpha)}\right.\right. \\
&\left.\left.-2^{\alpha} \frac{\Gamma(1+(s+1) \alpha)}{\Gamma(1+(s+2) \alpha)}+\frac{\Gamma(1+(s+1) \alpha)}{\Gamma(1+(s+3) \alpha)}\right]\left[\left|f^{(2 \alpha)}\left(a_{1}\right)\right|+\left|f^{(2 \alpha)}\left(a_{2}\right)\right|\right]\right\} . \\
& {[s(s)} \\
&
\end{aligned}
$$

This proves inequality (2.1). Since

$$
2^{\alpha(s-1)} f^{(2 \alpha)}\left(\frac{a_{1}+a_{2}}{2}\right) \leqslant \frac{\Gamma(1+s \alpha) \Gamma(1+\alpha)}{\Gamma(1+(s+1) \alpha)}\left(f^{(2 \alpha)}\left(a_{1}\right)+f^{(2 \alpha)}\left(a_{2}\right)\right),
$$

then

$$
\begin{aligned}
& \left|\frac{\Gamma(1+2 \alpha)}{2^{\alpha}} f\left(\frac{a_{1}+a_{2}}{2}\right)-\frac{\Gamma(1+2 \alpha)[\Gamma(1+\alpha)]^{2}}{2^{\alpha}\left(a_{2}-a_{1}\right)^{\alpha}} a_{1} I_{a_{2}}^{(\alpha)} f(x)\right| \\
& \quad \leqslant \frac{\left(a_{2}-a_{1}\right)^{2 \alpha}}{16^{\alpha}}\left\{\frac{2^{\alpha} \Gamma(1+(s+2) \alpha)}{\Gamma(1+(s+3) \alpha)}\left|f^{(2 \alpha)}\left(\frac{a_{1}+a_{2}}{2}\right)\right|+\left[\frac{\Gamma(1+s \alpha)}{\Gamma(1+(s+1) \alpha)}\right.\right.
\end{aligned}
$$




$$
\begin{aligned}
& \left.\left.-2^{\alpha} \frac{\Gamma(1+(s+1) \alpha)}{\Gamma(1+(s+2) \alpha)}+\frac{\Gamma(1+(s+2) \alpha)}{\Gamma(1+(s+3) \alpha)}\right]\left[\left|f^{(2 \alpha)}\left(a_{1}\right)\right|+\left|f^{(2 \alpha)}\left(a_{2}\right)\right|\right]\right\} \\
\leqslant & \frac{\left(a_{2}-a_{1}\right)^{2 \alpha}}{16^{\alpha}}\left\{\frac{2^{\alpha} \Gamma(1+(s+2) \alpha) 2^{-\alpha(s-1)} \Gamma(1+s \alpha) \Gamma(1+\alpha)}{\Gamma(1+(s+3) \alpha)}\left[\left|f^{(2 \alpha)}\left(a_{1}\right)\right|+\left|f^{(2 \alpha)}\left(a_{2}\right)\right|\right]\right. \\
& \left.+\left[\frac{\Gamma(1+s \alpha)}{\Gamma(1+(s+1) \alpha)}-\frac{2^{\alpha} \Gamma(1+(s+1) \alpha)}{\Gamma(1+(s+2) \alpha)}+\frac{\Gamma(1+(s+2) \alpha)}{\Gamma(1+(s+3) \alpha)}\right]\left[\left|f^{(2 \alpha)}\left(a_{1}\right)\right|+\left|f^{(2 \alpha)}\left(a_{2}\right)\right|\right]\right\} \\
= & \frac{\left(a_{2}-a_{1}\right)^{2 \alpha}}{16^{\alpha}}\left\{\frac{2^{\alpha(2-s)} \Gamma(1+(s+2) \alpha)}{\Gamma(1+(s+3) \alpha)} \frac{\Gamma(1+s \alpha) \Gamma(1+\alpha)}{\Gamma(1+(s+1) \alpha)}+\frac{\Gamma(1+s \alpha)}{\Gamma(1+(s+1) \alpha)}\right. \\
& \left.-\frac{2^{\alpha} \Gamma(1+(s+1) \alpha)}{\Gamma(1+(s+2) \alpha)}+\frac{\Gamma(1+(s+2) \alpha)}{\Gamma(1+(s+3) \alpha)}\left[\left|f^{(2 \alpha)}\left(a_{1}\right)\right|+\left|f^{(2 \alpha)}\left(a_{2}\right)\right|\right]\right\} .
\end{aligned}
$$

Thus, we get the inequality (2.2) and the proof is complete.

Remark 2.3.

1. When $\alpha=1$, Theorem 2.2 reduces to Theorem 2 in [15].

2. If $s=1$ in Theorem 2.2, then

$$
\begin{aligned}
& \left|\frac{\Gamma(1+2 \alpha)}{2^{\alpha}} f\left(\frac{a_{1}+a_{2}}{2}\right)-\frac{\Gamma(1+2 \alpha)[\Gamma(1+\alpha)]^{2}}{2^{\alpha}\left(a_{2}-a_{1}\right)^{\alpha}} a_{1} I_{a_{2}}^{(\alpha)} f(x)\right| \\
& \leqslant \frac{\left(a_{2}-a_{1}\right)^{2 \alpha}}{16^{\alpha}}\left\{\frac{2^{\alpha} \Gamma(1+3 \alpha)}{\Gamma(1+4 \alpha)}\left|f^{(2 \alpha)}\left(\frac{a_{1}+a_{2}}{2}\right)\right|+\left[\frac{\Gamma(1+\alpha)}{\Gamma(1+2 \alpha)}\right.\right. \\
& \left.\left.\quad-2^{\alpha} \frac{\Gamma(1+2 \alpha)}{\Gamma(1+3 \alpha)}+\frac{\Gamma(1+3 \alpha)}{\Gamma(1+4 \alpha)}\right]\left[\left|f^{(2 \alpha)}\left(a_{1}\right)\right|+\left|f^{(2 \alpha)}\left(a_{2}\right)\right|\right]\right\} \\
& \leqslant \\
& \quad \frac{\left(a_{2}-a_{1}\right)^{2 \alpha}}{16^{\alpha}}\left\{\frac{2^{\alpha} \Gamma(1+3 \alpha)}{\Gamma(1+4 \alpha)} \frac{[\Gamma(1+\alpha)]^{2}}{\Gamma(1+2 \alpha)}+\frac{\Gamma(1+\alpha)}{\Gamma(1+2 \alpha)}\right. \\
& \left.\quad-\frac{2^{\alpha} \Gamma(1+2 \alpha)}{\Gamma(1+3 \alpha)}+\frac{\Gamma(1+3 \alpha)}{\Gamma(1+4 \alpha)}\right\}\left[\left|f^{(2 \alpha)}\left(a_{1}\right)\right|+\left|f^{(2 \alpha)}\left(a_{2}\right)\right|\right] .
\end{aligned}
$$

3. If $s=1$ and $\alpha=1$ in Theorem 2.2 , then

$$
\begin{aligned}
\left|f\left(\frac{a_{1}+a_{2}}{2}\right)-\frac{1}{a_{2}-a_{1}} \int_{a_{1}}^{a_{2}} f(x) d x\right| & \leqslant \frac{\left(a_{2}-a_{1}\right)^{2}}{192}\left\{6\left|f^{\prime \prime}\left(\frac{a_{1}+a_{2}}{2}\right)\right|+\left|f^{\prime \prime}\left(a_{1}\right)\right|+\left|f^{\prime \prime}\left(a_{2}\right)\right|\right\} \\
& \leqslant \frac{\left(a_{2}-a_{1}\right)^{2}}{48}\left\{\left|f^{\prime \prime}\left(a_{1}\right)\right|+\left|f^{\prime \prime}\left(a_{2}\right)\right|\right\} .
\end{aligned}
$$

We give a new upper bound of the left generalized Hadamard's inequality for generalized s-convex functions in the following theorem:

Theorem 2.4. Assume that $\mathrm{f}: \mathrm{U} \subset[0, \infty) \longrightarrow \mathbb{R}^{\alpha}$ such that $\mathrm{f} \in \mathrm{D}_{\alpha}$ on $\operatorname{Int}(\mathrm{U})$ and $\mathrm{f}^{(2 \alpha)} \in \mathrm{C}_{\alpha}\left[\mathrm{a}_{1}, \mathrm{a}_{2}\right]$, where $\mathrm{a}_{1}, \mathrm{a}_{2} \in \mathrm{U}$ with $\mathrm{a}_{1}<\mathrm{a}_{1}$. If $\left|\mathrm{f}^{(2 \alpha)}\right|^{\mathrm{p}_{2}}$ is generalized s-convex on $\left[\mathrm{a}_{1}, \mathrm{a}_{2}\right]$ for some fixed $0<\mathrm{s} \leqslant 1$ and $\mathrm{p}_{2}>1$ with $\frac{1}{\mathrm{p}_{1}}+\frac{1}{\mathrm{p}_{2}}=1$, then the following inequality holds:

$$
\begin{aligned}
& \left|\frac{\Gamma(1+2 \alpha)}{2^{\alpha}} f\left(\frac{a_{1}+a_{2}}{2}\right)-\frac{\Gamma(1+2 \alpha)[\Gamma(1+\alpha)]^{2}}{2^{\alpha}\left(a_{2}-a_{1}\right)^{\alpha}} a_{1} I_{a_{2}}^{(\alpha)} f(x)\right| \\
& \leqslant \frac{\left(a_{2}-a_{1}\right)^{2 \alpha}}{16^{\alpha}}\left[\frac{\Gamma(1+s \alpha)}{\Gamma(1+(s+1) \alpha)}\right]^{\frac{1}{p_{2}}}\left[\frac{\Gamma\left(1+2 p_{1} \alpha\right)}{\Gamma\left(1+\left(2 p_{1}+1\right) \alpha\right)}\right]^{\frac{1}{p_{1}}} \\
& \quad \times\left[\left(\left|f^{(2 \alpha)}\left(\frac{a_{1}+a_{2}}{2}\right)\right|^{p_{2}}+\left|f^{(2 \alpha)}\left(a_{1}\right)\right|^{p_{2}}\right)^{\frac{1}{p_{2}}}+\left(\left|f^{(2 \alpha)}\left(\frac{a_{1}+a_{2}}{2}\right)\right|^{p_{2}}+\left|f^{(2 \alpha)}\left(a_{2}\right)\right|^{p_{2}}\right)^{\frac{1}{p_{2}}}\right] .
\end{aligned}
$$


Proof. Let $\mathrm{p}_{1}>1$, then from Lemma 2.1 and using generalized Hölder's inequality [20], we obtain

$$
\begin{aligned}
& \left|\frac{\Gamma(1+2 \alpha)}{2^{\alpha}} f\left(\frac{a_{1}+a_{2}}{2}\right)-\frac{\Gamma(1+2 \alpha)[\Gamma(1+\alpha)]^{2}}{2^{\alpha}\left(a_{2}-a_{1}\right)^{\alpha}} a_{1} I_{a_{2}}^{(\alpha)} f(x)\right| \\
& \leqslant \frac{\left(a_{2}-a_{1}\right)^{2 \alpha}}{16^{\alpha}}\left\{{ }_{0} I_{1}^{(\alpha)} \gamma^{2 \alpha}\left|f^{(2 \alpha)}\left(\gamma \frac{a_{1}+a_{2}}{2}+(1-\gamma) a_{1}\right)\right|\right. \\
& \left.\quad+I_{0}^{(\alpha)}(\gamma-1)^{2 \alpha}\left|f^{(2 \alpha)}\left(\gamma a_{2}+(1-\gamma) \frac{a_{1}+a_{2}}{2}\right)\right|\right\} \\
& \leqslant \\
& \quad \frac{\left(a_{2}-a_{1}\right)^{2 \alpha}}{16^{\alpha}}\left({ }_{0} I_{1}^{(\alpha)} \gamma^{2 p_{1} \alpha}\right)^{\frac{1}{p_{1}}}\left({ }_{0} I_{1}^{(\alpha)}\left|f^{(2 \alpha)}\left(\gamma \frac{a_{1}+a_{2}}{2}+(1-\gamma) a_{1}\right)\right|^{p_{2}}\right)^{\frac{1}{p_{2}}} \\
& \quad+\frac{\left(a_{2}-a_{1}\right)^{2 \alpha}}{16^{\alpha}}\left({ }_{0} I_{1}^{(\alpha)}(1-\gamma)^{2 p_{1} \alpha}\right)^{\frac{1}{p_{1}}}\left({ }_{0} I_{1}^{(\alpha)}\left|f^{(2 \alpha)}\left(\gamma a_{2}+(1-\gamma) \frac{a_{1}+a_{2}}{2}\right)\right|^{p_{2}}\right)^{\frac{1}{p_{2}}} .
\end{aligned}
$$

Since $\left|f^{(2 \alpha)}\right|^{p_{2}}$ is generalized s-convex, then

$$
\begin{aligned}
& { }_{0} \mathrm{I}_{1}^{(\alpha)}\left|f^{(2 \alpha)}\left(\gamma \frac{a_{1}+a_{2}}{2}+(1-\gamma) a_{1}\right)\right|^{p_{2}} \\
& \quad \leqslant \frac{\Gamma(1+s \alpha)}{\Gamma(1+(s+1) \alpha)}\left|f^{(2 \alpha)}\left(\frac{a_{1}+a_{2}}{2}\right)\right|^{p_{2}}+\frac{\Gamma(1+s \alpha)}{\Gamma(1+(s+1) \alpha)}\left|f^{(2 \alpha)}\left(a_{1}\right)\right|^{p_{2}}, \\
& { }_{0} I_{1}^{(\alpha)}\left|f^{(2 \alpha)}\left(\gamma a_{2}+(1-\gamma) \frac{a_{1}+a_{2}}{2}\right)\right|^{p_{2}} \\
& \quad \leqslant \frac{\Gamma(1+s \alpha)}{\Gamma(1+(s+1) \alpha)}\left|f^{(2 \alpha)}\left(a_{2}\right)\right|^{p_{2}}+\frac{\Gamma(1+s \alpha)}{\Gamma(1+(s+1) \alpha)}\left|f^{(2 \alpha)}\left(\frac{a_{1}+a_{2}}{2}\right)\right|^{p_{2}} .
\end{aligned}
$$

Hence

$$
\begin{aligned}
& \left|\frac{\Gamma(1+2 \alpha)}{2^{\alpha}} f\left(\frac{a_{1}+a_{2}}{2}\right)-\frac{\Gamma(1+2 \alpha)[\Gamma(1+\alpha)]^{2}}{2^{\alpha}\left(a_{2}-a_{1}\right)^{\alpha}} a_{1} I_{a_{2}}^{(\alpha)} f(x)\right| \\
& \leqslant \frac{\left(a_{2}-a_{1}\right)^{2 \alpha}}{16^{\alpha}}\left[\frac{\Gamma(1+s \alpha)}{\Gamma(1+(s+1) \alpha)}\right]^{\frac{1}{p_{2}}}\left[\frac{\Gamma\left(1+2 p_{1} \alpha\right)}{\Gamma\left(1+\left(2 p_{1}+1\right) \alpha\right)}\right]^{\frac{1}{p_{1}}} \\
& \quad \times\left\{\left[\left|f^{(2 \alpha)}\left(\frac{a_{1}+a_{2}}{2}\right)\right|^{p_{2}}+\left|f^{(2 \alpha)}\left(a_{1}\right)\right|^{p_{2}}\right]^{\frac{1}{p_{2}}}+\left[\left|f^{(2 \alpha)}\left(\frac{a_{1}+a_{2}}{2}\right)\right|^{p_{2}}+\left|f^{(2 \alpha)}\left(a_{2}\right)\right|^{p_{2}}\right]^{\frac{1}{p_{2}}}\right\} .
\end{aligned}
$$

The proof is complete.

Remark 2.5. If $s=1$ in Theorem 2.4, then

$$
\begin{aligned}
& \left|\frac{\Gamma(1+2 \alpha)}{2^{\alpha}} f\left(\frac{a_{1}+a_{2}}{2}\right)-\frac{\Gamma(1+2 \alpha)[\Gamma(1+\alpha)]^{2}}{2^{\alpha}\left(a_{2}-a_{1}\right)^{\alpha}} a_{1} I_{a_{2}}^{(\alpha)} f(x)\right| \\
& \leqslant \frac{\left(a_{2}-a_{1}\right)^{2 \alpha}}{16^{\alpha}}\left[\frac{\Gamma(1+\alpha)}{\Gamma(1+2 \alpha)}\right]^{\frac{1}{p_{2}}}\left[\frac{\Gamma\left(1+2 p_{1} \alpha\right)}{\Gamma\left(1+\left(2 p_{1}+1\right) \alpha\right)}\right]^{\frac{1}{p_{1}}} \\
& \quad \times\left\{\left[\left|f^{(2 \alpha)}\left(\frac{a_{1}+a_{2}}{2}\right)\right|^{p_{2}}+\left|f^{(2 \alpha)}\left(a_{1}\right)\right|^{p_{2}}\right]^{\frac{1}{p_{2}}}+\left[\left|f^{(2 \alpha)}\left(\frac{a_{1}+a_{2}}{2}\right)\right|^{p_{2}}+\left|f^{(2 \alpha)}\left(a_{2}\right)\right|^{p_{2}}\right]^{\frac{1}{p_{2}}}\right\} .
\end{aligned}
$$

Corollary 2.6. Assume that $\mathrm{f}: \mathrm{U} \subset[0, \infty) \longrightarrow \mathbb{R}^{\alpha}$ such that $\mathrm{f} \in \mathrm{D}_{\alpha}$ on $\operatorname{Int}(\mathrm{U})$ and $\mathrm{f}^{(2 \alpha)} \in \mathrm{C}_{\alpha}\left[\mathrm{a}_{1}, \mathrm{a}_{2}\right]$, where $\mathrm{a}_{1}, \mathrm{a}_{2} \in \mathrm{U}$ with $\mathrm{a}_{1}<\mathrm{a}_{1}$. If $\left|\mathrm{f}^{(2 \alpha)}\right|^{p_{2}}$ is generalized s-convex on $\left[\mathrm{a}_{1}, \mathrm{a}_{2}\right]$ for some fixed $0<\mathrm{s} \leqslant 1$ and $\mathrm{p}_{2}>1$ with $\frac{1}{\mathrm{p}_{1}}+\frac{1}{\mathrm{p}_{2}}=1$, then the following inequality holds:

$$
\left|\frac{\Gamma(1+2 \alpha)}{2^{\alpha}} f\left(\frac{a_{1}+a_{2}}{2}\right)-\frac{\Gamma(1+2 \alpha)[\Gamma(1+\alpha)]^{2}}{2^{\alpha}\left(a_{2}-a_{1}\right)^{\alpha}} a_{1} I_{a_{2}}^{(\alpha)} f(x)\right|
$$




$$
\begin{aligned}
\leqslant & \frac{\left(a_{2}-a_{1}\right)^{2 \alpha}}{16^{\alpha}} \frac{[\Gamma(1+s \alpha)]^{\frac{1}{p_{2}}}}{[\Gamma(1+(s+1) \alpha)]^{\frac{2}{p_{2}}}}\left[\frac{\Gamma\left(1+2 p_{1} \alpha\right)}{\Gamma\left(1+\left(2 p_{1}+1\right) \alpha\right)}\right]^{\frac{1}{p_{1}}}\left\{\left[\left(2^{\alpha(1-s)} \Gamma(1+s \alpha) \Gamma(1+\alpha)\right.\right.\right. \\
& \left.\left.+\Gamma(1+(s+1) \alpha))^{\frac{1}{p_{2}}}+2^{\frac{\alpha(1-s)}{p_{2}}}[\Gamma(1+\alpha)]^{\frac{1}{p_{2}}}[\Gamma(1+\alpha)]^{\frac{1}{p_{2}}}\right]\left[\left|f^{(2 \alpha)}\left(a_{1}\right)\right|+\left|f^{(2 \alpha)}\left(a_{2}\right)\right|\right]\right\} .
\end{aligned}
$$

Proof. Since $\left|\mathrm{f}^{(2 \alpha)}\right|^{\mathrm{p}_{2}}$ is generalized s-convex, then

$$
2^{\alpha(s-1)} f^{(2 \alpha)}\left(\frac{a_{1}+a_{2}}{2}\right) \leqslant \frac{\Gamma(1+s \alpha) \Gamma(1+\alpha)}{\Gamma(1+(s+1) \alpha)}\left(f^{(2 \alpha)}\left(a_{1}\right)+f^{(2 \alpha)}\left(a_{2}\right)\right)
$$

Hence using (2.3), we get

$$
\begin{aligned}
& \left|\frac{\Gamma(1+2 \alpha)}{2^{\alpha}} f\left(\frac{a_{1}+a_{2}}{2}\right)-\frac{\Gamma(1+2 \alpha)[\Gamma(1+\alpha)]^{2}}{2^{\alpha}\left(a_{2}-a_{1}\right)^{\alpha}} a_{1} I_{a_{2}}^{(\alpha)} f(x)\right| \\
& \leqslant \\
& \quad \frac{\left(a_{2}-a_{1}\right)^{2 \alpha}}{16^{\alpha}}\left[\frac{\Gamma(1+s \alpha)}{\Gamma(1+(s+1) \alpha)}\right]^{\frac{1}{p_{2}}}\left[\frac{\Gamma\left(1+2 p_{1} \alpha\right)}{\Gamma\left(1+\left(2 p_{1}+1\right) \alpha\right)}\right]^{\frac{1}{p_{1}}} \\
& \quad \times\left\{\left[2^{\alpha(1-s)} \frac{\Gamma(1+s \alpha) \Gamma(1+\alpha)}{\Gamma(1+(s+1) \alpha)}\left(\left|f^{(2 \alpha)}\left(a_{1}\right)\right|^{p_{2}}+\left|f^{(2 \alpha)}\left(a_{2}\right)\right|^{p_{2}}\right)+\left|f^{(2 \alpha)}\left(a_{1}\right)\right|^{p_{2}}\right]^{\frac{1}{p_{2}}}\right. \\
& \left.\quad+\left[2^{\alpha(1-s)} \frac{\Gamma(1+s \alpha) \Gamma(1+\alpha)}{\Gamma(1+(s+1) \alpha)}\left(\left|f^{(2 \alpha)}\left(a_{1}\right)\right|^{p_{2}}+\left|f^{(2 \alpha)}\left(a_{2}\right)\right|^{p_{2}}\right)+\left|f^{(2 \alpha)}\left(a_{2}\right)\right|^{p_{2}}\right]^{\frac{1}{p_{2}}}\right\} \\
& \leqslant \\
& \quad \frac{\left(a_{2}-a_{1}\right)^{2 \alpha}}{16^{\alpha}} \frac{[\Gamma(1+s \alpha)]^{\frac{1}{p_{2}}}}{[\Gamma(1+(s+1) \alpha)]^{\frac{2}{p_{2}}}}\left[\frac{\Gamma\left(1+2 p_{1} \alpha\right)}{\Gamma\left(1+\left(2 p_{1}+1\right) \alpha\right)}\right]^{\frac{1}{p_{1}}} \\
& \quad+\left\{\left[\left(2^{\alpha(1-s)} \Gamma(1+s \alpha) \Gamma(1+\alpha)+\Gamma(1+(s+1) \alpha)\right)\left|f^{(2 \alpha)}\left(a_{1}\right)\right|^{p_{2}}\right.\right. \\
& \left.\quad+2^{\alpha(1-s)} \Gamma(1+s \alpha) \Gamma(1+\alpha)\left|f^{(2 \alpha)}\left(a_{2}\right)\right|^{p_{2}}\right]^{\frac{1}{p_{2}}}+\left[2^{\alpha(1-s)} \Gamma(1+s \alpha) \Gamma(1+\alpha)\left|f^{(2 \alpha)}\left(a_{1}\right)\right|^{p_{2}}\right. \\
& \left.\quad+\left(2^{\alpha(1-s)} \Gamma(1+s \alpha) \Gamma(1+\alpha)+\Gamma(1+(s+1) \alpha)\right)\left|f^{(2 \alpha)}\left(a_{2}\right)\right|^{p_{2}}\right]
\end{aligned}
$$

and since $\sum_{i=1}^{k}\left(x_{i}+y_{i}\right)^{\alpha n} \leqslant \sum_{i=1}^{k} x_{i}^{\alpha n}+\sum_{i=1}^{k} y_{i}^{\alpha n}$ for $0<n<1, x_{i}, y_{i} \geqslant 0$, for all $1 \leqslant i \leqslant k$, then we have

$$
\begin{aligned}
& \left|\frac{\Gamma(1+2 \alpha)}{2^{\alpha}} f\left(\frac{a_{1}+a_{2}}{2}\right)-\frac{\Gamma(1+2 \alpha)[\Gamma(1+\alpha)]^{2}}{2^{\alpha}\left(a_{2}-a_{1}\right)^{\alpha}} a_{1} I_{a_{2}}^{(\alpha)} f(x)\right| \\
& \leqslant \frac{\left(a_{2}-a_{1}\right)^{2 \alpha}}{16^{\alpha}} \frac{[\Gamma(1+s \alpha)]^{\frac{1}{p_{2}}}}{[\Gamma(1+(s+1) \alpha)]^{\frac{2}{p_{2}}}}\left[\frac{\Gamma\left(1+2 p_{1} \alpha\right)}{\Gamma\left(1+\left(2 p_{1}+1\right) \alpha\right)}\right]^{\frac{1}{p_{1}}} \\
& \quad \times\left\{\left[\left(2^{\alpha(1-s)} \Gamma(1+s \alpha) \Gamma(1+\alpha)+\Gamma(1+(s+1) \alpha)\right)^{\frac{1}{p_{2}}}\left|f^{(2 \alpha)}\left(a_{1}\right)\right|\right.\right. \\
& \left.\quad+2^{\frac{\alpha(1-s)}{p_{2}}}[\Gamma(1+s \alpha)]^{\frac{1}{p_{2}}}[\Gamma(1+\alpha)]^{\frac{1}{p_{2}}}\left|f^{(2 \alpha)}\left(a_{2}\right)\right|\right] \\
& \quad+\left[2^{\frac{\alpha(1-s)}{p_{2}}}[\Gamma(1+s \alpha)]^{\frac{1}{p_{2}}}[\Gamma(1+\alpha)]^{\frac{1}{p_{2}}}\left|f^{(2 \alpha)}\left(a_{1}\right)\right|\right. \\
& \left.\left.\quad+\left(2^{\alpha(1-s)} \Gamma(1+s \alpha) \Gamma(1+\alpha)+\Gamma(1+(s+1) \alpha)\right)^{\frac{1}{p_{2}}}\left|f^{(2 \alpha)}\left(a_{2}\right)\right|\right]\right\},
\end{aligned}
$$

where $0<\frac{1}{p_{2}}<1$ for $p_{2}>1$. By a simple calculation, we obtain the required result. 
Now, the generalized Hadamard's type inequality for generalized s-concave functions.

Theorem 2.7. Assume that $\mathrm{f}: \mathrm{U} \subset[0, \infty) \longrightarrow \mathbb{R}^{\alpha}$ such that $\mathrm{f} \in \mathrm{D}_{\alpha}$ on $\operatorname{Int}(\mathrm{U})$ and $\mathrm{f}^{(2 \alpha)} \in \mathrm{C}_{\alpha}\left[\mathrm{a}_{1}, \mathrm{a}_{2}\right]$, where $\mathrm{a}_{1}, \mathrm{a}_{2} \in \mathrm{U}$ with $\mathrm{a}_{1}<\mathrm{a}_{1}$. If $\left|\mathrm{f}^{(2 \alpha)}\right|^{\mathrm{p}_{2}}$ is generalized s-convex on $\left[\mathrm{a}_{1}, \mathrm{a}_{2}\right]$ for some fixed $0<\mathrm{s} \leqslant 1$ and $\mathrm{p}_{2}>1$ with $\frac{1}{\mathrm{p}_{1}}+\frac{1}{\mathrm{p}_{2}}=1$, then the following inequality holds:

$$
\begin{aligned}
& \left|\frac{\Gamma(1+2 \alpha)}{2^{\alpha}} f\left(\frac{a_{1}+a_{2}}{2}\right)-\frac{\Gamma(1+2 \alpha)[\Gamma(1+\alpha)]^{2}}{2^{\alpha}\left(a_{2}-a_{1}\right)^{\alpha}} a_{1} I_{a_{2}}^{(\alpha)} f(x)\right| \\
& \leqslant \frac{2^{\frac{\alpha(s-1)}{p_{2}}}\left(a_{2}-a_{1}\right)^{2 \alpha}}{16^{\alpha}(\Gamma(1+\alpha))^{\frac{1}{p_{2}}}}\left[\frac{\Gamma\left(1+2 p_{1} \alpha\right)}{\Gamma\left(1+\left(2 p_{1}+1\right) \alpha\right)}\right]^{\frac{1}{p_{1}}}\left[\left|f^{(2 \alpha)}\left(\frac{3 a_{1}+a_{2}}{4}\right)\right|+\left|f^{(2 \alpha)}\left(\frac{a_{1}+3 a_{2}}{4}\right)\right|\right] .
\end{aligned}
$$

Proof. From Lemma 2.1 and using the generalized Hölder inequality for $p_{2}>1$ and $\frac{1}{p_{1}}+\frac{1}{p_{2}}=1$, we get

$$
\begin{aligned}
& \left|\frac{\Gamma(1+2 \alpha)}{2^{\alpha}} f\left(\frac{a_{1}+a_{2}}{2}\right)-\frac{\Gamma(1+2 \alpha)[\Gamma(1+\alpha)]^{2}}{2^{\alpha}\left(a_{2}-a_{1}\right)^{\alpha}}{ }_{a_{1}} I_{a_{2}}^{(\alpha)} f(x)\right| \\
& \leqslant \\
& \quad \frac{\left(a_{2}-a_{1}\right)^{2 \alpha}}{16^{\alpha}}\left[{ }_{0} I_{1}^{(\alpha)} \gamma^{2 \alpha}\left|f^{(2 \alpha)}\left(\gamma \frac{a_{1}+a_{2}}{2}+(1-\gamma) a_{1}\right)\right|\right. \\
& \left.\quad+{ }_{0} I_{1}^{(\alpha)}(\gamma-1)^{2 \alpha}\left|f^{(2 \alpha)}\left(\gamma a_{2}+(1-\gamma) \frac{a_{1}+a_{2}}{2}\right)\right|\right] \\
& \leqslant \\
& \quad \frac{\left(a_{2}-a_{1}\right)^{2 \alpha}}{16^{\alpha}}\left({ }_{0} I_{1}^{(\alpha)} \gamma^{2 p_{1} \alpha}\right)^{\frac{1}{p_{1}}}\left({ }_{0} I_{1}^{(\alpha)}\left|f^{(2 \alpha)}\left(\gamma \frac{a_{1}+a_{2}}{2}+(1-\gamma) a_{1}\right)\right|^{p_{2}}\right)^{\frac{1}{p_{2}}} \\
& \quad+\frac{\left(a_{2}-a_{1}\right)^{2 \alpha}}{16^{\alpha}}\left({ }_{0} I_{1}^{(\alpha)}(\gamma-1)^{2 p_{1} \alpha}\right)^{\frac{1}{p_{1}}}\left({ }_{0} I_{1}^{(\alpha)}\left|f^{(2 \alpha)}\left(\gamma a_{2}+(1-\gamma) \frac{a_{1}+a_{2}}{2}\right)\right|^{p_{2}}\right)^{\frac{1}{p_{2}}} .
\end{aligned}
$$

Since $\left|f^{(2 \alpha)}\right|^{p_{2}}$ is generalized s-concave, then

$$
{ }_{0} \mathrm{I}_{1}^{(\alpha)}\left|\mathrm{f}^{(2 \alpha)}\left(\gamma \frac{\mathrm{a}_{1}+\mathrm{a}_{2}}{2}+(1-\gamma) \mathrm{a}_{1}\right)\right|^{\mathrm{p}_{2}} \leqslant \frac{2^{\alpha(s-1)}}{\Gamma(1+\alpha)}\left|\mathrm{f}^{(2 \alpha)}\left(\frac{3 \mathrm{a}_{1}+\mathrm{a}_{2}}{4}\right)\right|^{p_{2}},
$$

also

$$
{ }_{0} I_{1}^{(\alpha)}\left|f^{(2 \alpha)}\left(\gamma a_{2}+(1-\gamma) \frac{a_{1}+a_{2}}{2}\right)\right|^{p_{2}} \leqslant \frac{2^{\alpha(s-1)}}{\Gamma(1+\alpha)}\left|f^{(2 \alpha)}\left(\frac{a_{1}+3 a_{2}}{4}\right)\right|^{p_{2}} .
$$

From (2.5) and (2.6), we observe that

$$
\begin{aligned}
& \left|\frac{\Gamma(1+2 \alpha)}{2^{\alpha}} f\left(\frac{a_{1}+a_{2}}{2}\right)-\frac{\Gamma(1+2 \alpha)[\Gamma(1+\alpha)]^{2}}{2^{\alpha}\left(a_{2}-a_{1}\right)^{\alpha}} a_{1} I_{a_{2}}^{(\alpha)} f(x)\right| \\
& \leqslant \frac{\left(a_{2}-a_{1}\right)^{2 \alpha}}{16^{\alpha}}\left[\frac{\Gamma\left(1+2 p_{1} \alpha\right)}{\Gamma\left(1+\left(2 p_{1}+1\right) \alpha\right)}\right]^{\frac{1}{p_{1}}} \frac{2^{\frac{\alpha(s-1)}{p_{2}}}}{(\Gamma(1+\alpha))^{\frac{1}{p_{2}}}}\left|f^{(2 \alpha)}\left(\frac{3 a_{1}+a_{2}}{4}\right)\right| \\
& \quad+\frac{\left(a_{2}-a_{1}\right)^{2 \alpha}}{16^{\alpha}}\left[\frac{\Gamma\left(1+2 p_{1} \alpha\right)}{\Gamma\left(1+\left(2 p_{1}+1\right) \alpha\right)}\right]^{\frac{1}{p_{1}}} \frac{2^{\frac{\alpha(s-1)}{p_{2}}}}{(\Gamma(1+\alpha))^{\frac{1}{p_{2}}}}\left|f^{(2 \alpha)}\left(\frac{a_{1}+3 a_{2}}{4}\right)\right| \\
& =\frac{2^{\frac{\alpha(s-1)}{p_{2}}}\left(a_{2}-a_{1}\right)^{2 \alpha}}{16^{\alpha}(\Gamma(1+\alpha))^{\frac{1}{p_{2}}}}\left[\frac{\Gamma\left(1+2 p_{1} \alpha\right)}{\Gamma\left(1+\left(2 p_{1}+1\right) \alpha\right)}\right]^{\frac{1}{p_{1}}}\left[\left|f^{(2 \alpha)}\left(\frac{3 a_{1}+a_{2}}{4}\right)\right|+\left|f^{(2 \alpha)}\left(\frac{a_{1}+3 a_{2}}{4}\right)\right|\right] .
\end{aligned}
$$

So, the proof is complete. 
Remark 2.8.

1. If $\alpha=1$ in Theorem 2.7, then

$$
\begin{aligned}
& \left|f\left(\frac{a_{1}+a_{2}}{2}\right)-\frac{1}{a_{2}-a_{1}} \int_{a_{1}}^{a_{2}} f(x) d x\right| \\
& \quad \leqslant \frac{2^{\frac{s-1}{q}}\left(a_{2}-a_{1}\right)^{2}}{16}\left[\frac{1}{\Gamma\left(2 p_{1}+1\right)}\right]^{\frac{1}{p_{1}}}\left[\left|f^{\prime \prime}\left(\frac{3 a_{1}+a_{2}}{4}\right)\right|+\left|f^{\prime \prime}\left(\frac{a_{1}+3 a_{2}}{4}\right)\right|\right] .
\end{aligned}
$$

2. If $s=1$ and $\frac{1}{3}<\left[\frac{\Gamma\left(1+2 \mathrm{p}_{1} \alpha\right)}{\Gamma\left(1+\left(2 \mathrm{p}_{1}+1\right) \alpha\right)}\right]^{\frac{1}{p_{1}}}<1, \mathrm{p}_{1}>1$ in Theorem 2.7, then

$$
\begin{aligned}
& \left|\frac{\Gamma(1+2 \alpha)}{2^{\alpha}} f\left(\frac{a_{1}+a_{2}}{2}\right)-\frac{\Gamma(1+2 \alpha)[\Gamma(1+\alpha)]^{2}}{2^{\alpha}\left(a_{2}-a_{1}\right)^{\alpha}} a_{1} I_{a_{2}}^{(\alpha)} f(x)\right| \\
& \quad \leqslant \frac{\left(a_{2}-a_{1}\right)^{2 \alpha}}{16^{\alpha}(\Gamma(1+\alpha))^{\frac{1}{p_{2}}}}\left[\left|f^{(2 \alpha)}\left(\frac{3 a_{1}+a_{2}}{4}\right)\right|+\left|f^{(2 \alpha)}\left(\frac{a_{1}+3 a_{2}}{4}\right)\right|\right] .
\end{aligned}
$$

\section{Applications to special means}

As in [16], some generalized means are considered such as:

$$
\begin{aligned}
A\left(a_{1}, a_{2}\right) & =\frac{a_{1}^{\alpha}+a_{2}^{\alpha}}{2^{\alpha}}, a_{1}, a_{2} \geqslant 0, \\
L_{n}\left(a_{1}, a_{2}\right) & =\left[\frac{\Gamma(1+n \alpha)}{\Gamma(1+(n+1) \alpha)}\left(a_{2}^{(n+1) \alpha}-a_{1}^{(n+1) \alpha}\right)\right]^{\frac{1}{n}}, n \in \mathbb{Z}\{-1,0\}, a_{1}, a_{2} \in \mathbb{R}, a_{1} \neq a_{2} .
\end{aligned}
$$

In [12], the following example was given:

let $0<s<1$ and $a_{1}^{\alpha}, a_{2}^{\alpha}, a_{3}^{\alpha} \in \mathbb{R}^{\alpha}$. Define for $x \in \mathbb{R}_{+}$,

$$
f(n)= \begin{cases}a_{1}^{\alpha}, & n=0 \\ a_{2}^{\alpha} n^{s \alpha}+a_{3}^{\alpha}, & n>0\end{cases}
$$

If $a_{2}^{\alpha} \geqslant 0^{\alpha}$ and $0^{\alpha} \leqslant a_{3}^{\alpha} \leqslant a_{1}^{\alpha}$, then $f \in G_{s}^{2}$.

Proposition 3.1. Let $0<a_{1}<a_{2}$ and $s \in(0,1)$. Then

$$
\begin{aligned}
& \left|\frac{\Gamma(1+2 \alpha)}{2^{\alpha}} A^{s}\left(a_{1}, a_{2}\right)-\frac{\Gamma(1+2 \alpha)[\Gamma(1+\alpha)]^{2}}{2^{\alpha}\left(a_{2}-a_{1}\right)^{\alpha}} L_{s}^{s}\left(a_{1}, a_{2}\right)\right| \\
& \leqslant \frac{\left(a_{2}-a_{1}\right)^{2 \alpha}}{16^{\alpha}}\left|\frac{\Gamma(1+s \alpha)}{\Gamma(1+(s-2) \alpha)}\right|\left\{\frac{2^{\alpha} \Gamma(1+3 \alpha)[\Gamma(1+\alpha)]^{2}}{\Gamma(1+4 \alpha) \Gamma(1+2 \alpha)}\right. \\
& \left.\quad+\frac{\Gamma(1+\alpha)}{\Gamma(1+2 \alpha)}-\frac{2^{\alpha} \Gamma(1+2 \alpha)}{\Gamma(1+3 \alpha)}+\frac{\Gamma(1+3 \alpha)}{\Gamma(1+4 \alpha)}\right\}\left[\left|a_{1}\right|^{(s-2) \alpha}+\left|a_{2}\right|^{(s-2) \alpha}\right] .
\end{aligned}
$$

Proof. The result follows from Remark 2.3 (2) with $f:[0,1] \longrightarrow\left[0^{\alpha}, 1^{\alpha}\right], f(x)=x^{s \alpha}$. Also, when $\alpha=1$, we have the following inequalitly:

$$
\left|A^{s}\left(a_{1}, a_{2}\right)-\frac{1}{a_{2}-a_{1}} L_{s}^{s}\left(a_{1}, a_{2}\right)\right| \leqslant \frac{\left(a_{2}-a_{1}\right)^{2}|s(s-1)|}{48}\left\{\left|a_{1}\right|^{s-2}+\left|a_{2}\right|^{s-2}\right\} .
$$


Proposition 3.2. Let $0<\mathrm{a}_{1}<\mathrm{a}_{2}$ and $\mathrm{s} \in(0,1)$. Then

$$
\begin{aligned}
& \left|\frac{\Gamma(1+2 \alpha)}{2^{\alpha}} A^{s}\left(a_{1}, a_{2}\right)-\frac{\Gamma(1+2 \alpha)[\Gamma(1+\alpha)]^{2}}{2^{\alpha}\left(a_{2}-a_{1}\right)^{\alpha}} L_{s}^{s}\left(a_{1}, a_{2}\right)\right| \\
& \leqslant \frac{\left(a_{2}-a_{1}\right)^{2 \alpha}}{16^{\alpha}}\left[\frac{\Gamma(1+\alpha)}{\Gamma(1+2 \alpha)}\right]^{\frac{1}{p_{2}}}\left|\frac{\Gamma(1+s \alpha)}{\Gamma(1+(s-2) \alpha)}\right|\left[\frac{\Gamma\left(1+2 p_{1} \alpha\right)}{\Gamma\left(1+\left(2 p_{1}+1\right) \alpha\right)}\right]^{\frac{1}{p_{1}}} \\
& \quad \times\left[\left(\left|\frac{a_{1}+a_{2}}{2}\right|^{(s-2) p_{2} \alpha}+\left|a_{1}\right|^{(s-2) p_{2} \alpha}\right)^{\frac{1}{p_{2}}}+\left(\left|\frac{a_{1}+a_{2}}{2}\right|^{(s-2) p_{2} \alpha}+\left|a_{2}\right|^{(s-2) p_{2} \alpha}\right)^{\frac{1}{p_{2}}}\right],
\end{aligned}
$$

where $\mathrm{p}_{2}>1$ and $\frac{1}{\mathrm{p}_{1}}+\frac{1}{\mathrm{p}_{2}}=1$.

Proof. The result follows (2.4) with $f:[0,1] \longrightarrow\left[0^{\alpha}, 1^{\alpha}\right], f(x)=x^{s \alpha}$ and when $\alpha=1$, we have the following inequalitly:

$$
\begin{aligned}
& \left|A^{s}\left(a_{1}, a_{2}\right)-\frac{1}{a_{2}-a_{1}} L_{s}^{s}\left(a_{1}, a_{2}\right)\right| \leqslant \frac{\left(a_{2}-a_{1}\right)^{2}|s(s-1)|}{2^{\frac{1}{p_{2}}} 16\left(2 p_{1}+1\right)^{\frac{1}{p_{1}}}}\left\{\left(\left|\frac{a_{1}+a_{2}}{2}\right|^{(s-2) p_{2}}+\left|a_{1}\right|^{(s-2) p_{2}}\right)^{\frac{1}{p_{2}}}\right. \\
& \left.+\left(\left|\frac{a_{1}+a_{2}}{2}\right|^{(s-2) p_{2}}+\left|a_{2}\right|^{(s-2) p_{2}}\right)^{\frac{1}{p_{2}}}\right\} \text {. }
\end{aligned}
$$

Where $A\left(a_{1}, a_{2}\right)$ and $L_{n}\left(a_{1}, a_{2}\right)$ in (3.1) and (3.2) are known as

1. arithmetic mean: $A\left(a_{1}, a_{2}\right)=\frac{a_{1}+a_{2}}{2}, a_{1}, a_{2} \in \mathbb{R}^{+}$;

2. logarithmic mean: $L\left(a_{1}, a_{2}\right)=\frac{a_{1}-a_{2}}{\ln \left|a_{1}\right|-\ln \mid a_{2}},\left|a_{1}\right| \neq a_{2}, a_{1}, a_{2} \neq 0, a_{1}, a_{2} \in \mathbb{R}^{+}$;

3. generalized Log-mean: $L_{n}\left(a_{1}, a_{2}\right)=\left[\frac{a_{2}^{n+1}-a_{1}^{n+1}}{(n+1)\left(a_{2}-a_{1}\right)}\right]^{\frac{1}{n}}, n \in \mathbb{Z} \backslash\{-1,0\}, a_{1}, a_{2} \in \mathbb{R}^{+}$.

Now, we give application to wave equation on Cantor sets:

Not only the fractional calculus which deals with fractional differential equations [11] has various kinds of analytical methods for solving these equations [1, 2], but also local fractional calculus which deals with problems for nondifferentiable function [19] has analytical methods for solving the local fractional differential equations such as local fractional Fourier series method was applied to process the wave equation on Cantor sets (local fractional wave equation) with local fractional derivatives [20].

The wave equation on Cantor sets was given as

$$
\frac{\partial^{2 \alpha} f(x, t)}{\partial t^{2 \alpha}}=A^{2 \alpha} \frac{\partial^{2 \alpha} f(x, t)}{\partial x^{2 \alpha}}
$$

Following (3.3), a wave equation on Cantor sets was proposed as follows [22]:

$$
\frac{\partial^{2 \alpha} f(x, t)}{\partial t^{2 \alpha}}=\frac{x^{2 \alpha}}{\Gamma(1+2 \alpha)} \frac{\partial^{2 \alpha} f(x, t)}{\partial x^{2 \alpha}}, \quad 0 \leqslant \alpha \leqslant 1,
$$

where $f(x, t)$ is a fractal wave function and the initial value is given by $f(x, 0)=\frac{x^{\alpha}}{\Gamma(1+\alpha)}$. The solution of (3.4) is given as $f(x, t)=\frac{x^{\alpha}}{\Gamma(1+\alpha)}+\frac{t^{2 \alpha}}{\Gamma(1+2 \alpha)}$.

By using Lemma 2.1, we have

$$
\frac{\Gamma(1+2 \alpha) \Gamma(1+\alpha)}{2^{\alpha}\left(a_{2}-a_{1}\right)^{\alpha}} \int_{a_{1}}^{a_{2}} f(x, t)(d t)^{\alpha}-\frac{\Gamma(1+2 \alpha)}{2^{\alpha}} f\left(x, \frac{a_{1}+a_{2}}{2}\right)
$$




$$
\begin{aligned}
= & \frac{\left(a_{2}-a_{1}\right)^{\alpha}}{8^{\alpha} \Gamma(1+2 \alpha)}\left[\left(\frac{2}{a_{2}-a_{1}}\right)^{2 \alpha} a_{1} I_{\frac{a_{2}+a_{1}}{2}}^{(\alpha)}\left(t-a_{1}\right)^{2 \alpha} x^{2 \alpha} \frac{\partial^{2 \alpha} f(x, t)}{\partial x^{\alpha}}\right. \\
& \left.+a_{1} I_{\frac{a_{2}+a_{1}}{2}}^{(\alpha)}\left(\frac{2\left(t-a_{1}\right)}{a_{2}-a_{1}}-1\right)^{2 \alpha} x^{2 \alpha} \frac{\partial^{2 \alpha} f(x, t)}{\partial x^{\alpha}}\right] .
\end{aligned}
$$

\section{Conclusion}

In this article, we have studied some generalized Hermite-Hadamard type inequalities for the class of functions whose second local fractional derivatives in absolute value at certain powers are generalized s-convex functions in the second sense on fractal sets. In particular, our results extend some important inequalities in classical situation and, when $\alpha=1$, some relationships between these inequalities and the classical inequalities have been established. Finally, we have given some applications for these inequalities on fractal sets.

\section{Acknowledgment}

The authors are exceptionally grateful to the anonymous referees for their valuable suggestions and comments, which helped the authors to improve the work.

\section{References}

[1] A. Atangana, S. B. Belhaouari, Solving partial differential equation with space- and time-fractional derivatives via homotopy decomposition method, Math. Probl. Eng., 2013 (2013), 9 pages. 3

[2] A. Atangana, E. F. Doungmo-Goufo, Solution of diffusion equation with local derivative with new parameter, Therm. Sci., 19 (2015), 231-238. 3

[3] D. Baleanu, H. M. Srivastava, X.-J. Yang, Local fractional variational iteration algorithms for the parabolic Fokker-Planck equation defined on Cantor sets, Prog. Fract. Differ. Appl., 1 (2015), 1-11. 1

[4] S. S. Dragomir, On the Hadamard's inequality for convex functions on the co-ordinates in a rectangle from the plane, Taiwanese J. Math., 5 (2001), 775-788. 1

[5] S. S. Dragomir, S. Fitzpatrick, The Hadamard inequalities for s-convex functions in the second sense, Demonstratio Math., 32 (1999), 687-696. 1, 1

[6] M. Grinblatt, J. T. Linnainmaa, Jensen's inequality, parameter uncertainty, and multi-period investment, Rev. Asset Pric. Stud., 1 (2011), 1-34. 1

[7] L. Hörmander, Notions of convexity, Progress in Mathematics, Birkhäuser Boston, Inc., Boston, MA, (1994). 1

[8] J. Hua, B.-Y. Xi, F. Qi, Inequalities of Hermite-Hadamard type involving an s-convex function with applications, Appl. Math. Comput., 246 (2014), 752-760. 1

[9] A. Kilıçman, W. Saleh, Notions of generalized s-convex functions on fractal sets, J. Inequal. Appl., 2015 (2015), 16 pages. 1

[10] A. Kılıçman, W. Saleh, Some generalized Hermite-Hadamard type integral inequalities for generalized s-convex functions on fractal sets, Adv. Difference Equ., 2015 (2015), 15 pages. 1

[11] A. A. Kilbas, H. M. Srivastava, J. J. Trujillo, Theory and applications of fractional differential equations, North-Holland Mathematics Studies, Elsevier Science B.V., Amsterdam, (2006). 3

[12] H.-X. Mo, X. Sui, Generalized s-convex functions on fractal sets, Abstr. Appl. Anal., 2014 (2014), 8 pages . 1, 3

[13] H.-X. Mo, X. Sui, Hermite-Hadamard type inequalities for generalized s-convex functions on real linear fractal set $\mathbb{R}^{\alpha}(0<$ $<1$ ), ArXiv, 2015 (2015), 10 pages. 1

[14] H.-X. Mo, X. Sui, D.-Y. Yu, Generalized convex functions on fractal sets and two related inequalities, Abstr. Appl. Anal., 2014 (2014), 7 pages. 1, 1

[15] M. E. Özdemir, Ç. Yıldız, A. O. Akdemir, E. Set, On some inequalities for s-convex functions and applications, J. Inequal. Appl., 2013 (2013), 11 pages. 1

[16] C. E. M. Pearce, J. Pečarić, Inequalities for differentiable mappings with application to special means and quadrature formulx, Appl. Math. Lett., 13 (2000), 51-55. 3

[17] J. J. Ruel, M. P. Ayres, Jensen's inequality predicts effects of environmental variation, Trends Ecol. Evol., 14 (1999), 361-366. 1

[18] M. Z. Sarikaya, T. Tunc, H. Budak, On generalized some integral inequalities for local fractional integrals, Appl. Math. Comput., 276 (2016), 316-323. 1

[19] X.-J. Yang, Local fractional integral transforms, Prog. Nonlinear Sci., 4 (2011), 1-225. 3 
[20] X.-J. Yang, Advanced local fractional calculus and its applications, World Science Publ., New York, (2012). 2, 3

[21] X.-J. Yang, D. Baleanu, H. M. Srivastava, Local fractional similarity solution for the diffusion equation defined on Cantor sets, Appl. Math. Lett., 47 (2015), 54-60. 1

[22] A.-M. Yang, X.-J. Yang, Z.-B. Li, Local fractional series expansion method for solving wave and diffusion equations on Cantor sets, Abstr. Appl. Anal., 2013 (2013), 5 pages. 3 